\title{
Radial and Tangential Winding Coil Probes for Sextupole Magnet Measurements
}

\section{Introduction}

Rotating coil probes of radial and tangential winding geometries for the measurements of the magnetic center, main field integral and multipole coefficients of sextupole magnets are described. Two sets of coils are sufficient for a probe of radial winding geometry. For a tangential winding probe, however, typically several sets of coils are required to measure the above magnetic parameters. The tangential coil geometry in this note is described with three sets of coils. The main sextupole field coefficients are defined $a s b_{2}=1.0 \mathrm{~cm}^{-2}$ and $a_{2}=0$ for the expression of the multipole field coefficients Eq. (1) in Reference 1.

\section{Radial Winding Coils}

The flux linkage for the two coils in Fig. 1, coil A and coil B, is given in Eqs. (5) and (6) in Reference 1 and is written here again,

$$
\begin{aligned}
& \phi_{A B}(\theta)=L N_{A} \sum_{n=1}^{\infty}\left(C_{n} / R^{n-1}\right)\left(r_{A 2} n / n\right) R_{f a c} \operatorname{cosn}\left(\theta-\alpha_{n}\right), \\
& R_{f a c}=1-\left(-r_{A 1} / r_{A 2}\right)^{n}-\left(N_{B} / N_{A}\right)\left[r_{B 2} n-\left(-r_{B 1}\right)^{n}\right] / r_{A 2} n,
\end{aligned}
$$

where $N_{A}$ and $N_{B}$ are the number of turns of coil $A$ and coil $B$, respectively. From Eq. (2) the conditions for the rejection of the quadrupole and sextupole components for the measurements of higher multipole coefficients, $n \geq 4$, are given by

$$
\begin{aligned}
& \left(r_{A 2}{ }^{2}-r_{A 1}^{2}\right)-\left(N_{B} / N_{A}\right)\left(r_{B 2}{ }^{2}-r_{B 1}^{2}\right)=0, \\
& {\left[r_{A 2}{ }^{3}-\left(-r_{A 1}\right)^{3}\right]-\left(N_{B} / N_{A}\right)\left[r_{B 2}^{3}-\left(-r_{B 1}\right)^{3}\right]=0 .}
\end{aligned}
$$




\section{DISCLAIMER}

Portions of this document may be illegible in electronic image products. Images are produced from the best available original document. 
By introducing the following parameters

$$
\begin{array}{ll}
R=\left(r_{A 2}-r_{A 1}\right) / 2, & r=\left(r_{B 2}-r_{B 1}\right) / 2, \\
C=\left(r_{A 2}+r_{A 1}\right) / 2, & D=\left(r_{B 2}+r_{B 1}\right) / 2,
\end{array}
$$

the above two conditions, Eqs. (3) and (4), are transformed into

$$
\begin{aligned}
& R C=\left(N_{B} / N_{A}\right) r D \\
& \left(R^{2}+3 C^{2}\right) / C=\left(x^{2}+3 D^{2}\right) / D .
\end{aligned}
$$

By solving Eq. (6), coil parameters for $\mathrm{N}_{B} / \mathrm{N}_{A}=3.5$ as an example, are listed in Table 1.

When the cylinder rotation axis (CR) in Fig. 1 is displaced from the magnetic center by

$$
z_{0}=r_{0} \exp \left(i \theta_{0}\right)
$$

it is shown in Eq. (10) Reference 1 that for a typical sextupole magnet, $C_{n}(n \neq 3) / C_{3}<1$; the quadrupole and sextupole components are given by

$$
B_{\theta}^{\prime}+i B_{I}^{\prime}=\left(C_{3} / R^{2}\right) \exp \left(-i 3 \alpha_{3}\right)\left(z_{0}+z^{\prime}\right)^{2} .
$$

Here $z^{\prime}=r^{\prime} \exp \left(i \theta^{\prime}\right)$ is a position on the surface of the rotating coil cylinder at $r^{\prime}=r_{A 2}$. The flux linkage for coil A is calculated from Eq. (8),

$$
\begin{aligned}
\phi_{\mathrm{A}}^{\prime}\left(\theta^{\prime}\right)=\operatorname{LN}_{\mathrm{A}}\left(C_{3} / R^{2}\right) & {\left[r_{0}\left(r_{A 2}{ }^{2}-r_{A 1}^{2}\right) \cos \left(2 \theta^{\prime}+\theta_{0}-3 \alpha_{3}\right)\right.} \\
& \left.\frac{r_{A 2}{ }^{3}-\left(-r_{A 1}\right)^{3}}{3} \cos 3\left(\theta^{\prime}-\alpha_{3}\right)\right] .
\end{aligned}
$$


From Eq. (9) the $\cos 2 \theta^{3}$ term, which is the quadrupole field component due to the displacement of the magnetic center by Eq. (7), measures the magnetic center and the $\cos 3 \theta^{\prime}$ term measures the sextupole field integral. It should be noted that in Eq. (8) there is a dipole field component term which is proportional to $\mathrm{r}_{0}{ }^{2}$ and negligible. This is why the large sensitivity for $n=1$ in Table 1 is not important. This is also why finding the magnetic center for a sextupole magnet is defined for the minimization of the quadrupole field component instead of that of the dipole field component in this note.

When the conductor positions of $r_{A 2}$ and $r_{A 1}$, and the coil plane have errors of $\Delta r_{A}$, as shown in Fig. 3 in Reference 1, Eq. (9) has additional terms. The corresponding expression with correction terms is

$$
\phi_{A}^{\prime}\left(\theta^{\prime}\right)=\operatorname{LN}_{A}\left(C_{3} / R^{2}\right)\left(x_{0} Q+S\right),
$$

with

$$
\begin{aligned}
& Q=\left(r_{A 2}{ }^{2}-r_{A 1}{ }^{2}\right)\left(1+2 \frac{\Delta r_{A}}{r_{A 2}}\right) \cos \left(2 \theta^{\prime}+\theta_{0}-3 \alpha_{3}\right)-3 \Delta r_{A} \cdot r_{A 1} \sin \left(2 \theta^{\prime}+\theta_{0}-3 \alpha_{3}\right), \\
& S=\frac{r_{A 2}{ }^{3}-\left(-r_{A 1}\right)^{3}}{3}\left(1+\frac{\Delta r_{A A}}{r_{A 2}} \cos 3\left(\theta^{\prime}-\alpha_{3}\right)+\frac{3}{2} \Delta r_{A} \cdot r_{A 1}{ }^{2} \sin 3\left(\theta^{\prime}-\alpha_{3}\right),\right.
\end{aligned}
$$

where it is assumed that $r_{A 2}=2 r_{A 1}$, and the position of $r_{A 2}$ is the reference angular position. The correction terms are measured from the real and imaginary parts of Fourier analysis.

\section{Tangential Winding Coil}

Figure 2 shows the cross section of a tangential winding coil geometry with one $\Delta$-coil and two $\pi / 2$-coils. The two $\pi / 2$-coils do have both roles for the $\pi / 2$-coils and $\pi / 3$-coils (see Reference 2 ) by series-connections of the two coils; one connection adds up the quadrupole field component and one connection adds up the main sextupole field components. The two series-connected coils will be called $Q Q$ and $Q S$ coils, and will have different numbers of turns of the coils, $N_{A}$ and $N_{B}$ respectively. Finally the three coils, $\Delta$-coil, $Q Q$-coil and $Q S$-coil, are connected to have the flux linkage such that 


$$
\begin{aligned}
& \phi_{\Delta}(\theta)-\phi_{Q Q}(\theta)-\phi_{Q S}(\theta)={ }^{L N}{ }_{\Delta} \sum_{n=1}^{\infty}\left(C_{n} / R^{n-1}\right) \frac{r^{n}}{n} 2 \sin n\left(\theta+\frac{\pi}{2}-\alpha_{n}\right) * \\
& {\left[\sin \left(\frac{n \Delta}{2}\right)-\left\{1+(-1)^{n}\right\} \frac{N_{Q Q}}{N_{\Delta}} \sin \left(\frac{n \pi}{4}\right)-\left\{1-(-1)^{n}\right\} \frac{{ }_{Q S}^{N}}{N_{\Delta}} \sin \left(\frac{n \pi}{4}\right) .\right.}
\end{aligned}
$$

From Eq. (11) the conditions for the rejection of the quadrupole and sextupole components are

$$
\begin{aligned}
& C_{2}\left[{ }^{\mathrm{N}} \Delta \sin (\Delta)-2 \mathrm{~N}_{Q Q}\right]=0, \\
& \mathrm{C}_{3}\left[{ }^{\mathrm{N}}{ }_{\Delta} \sin (3 \Delta / 2)-2 \mathrm{~N}_{Q S} \sin (3 \pi / 4)\right]=0
\end{aligned}
$$

With a choice of the tangential coil parameters,

$$
\mathrm{N}_{\Delta}=14, \mathrm{~N}_{Q Q}=2, \mathrm{~N}_{Q S}=4 \text { and } \Delta=15.3882^{\circ} \text {, }
$$

relative sensitivities of the multipole coefficients are listed in Table 2.

For a sextupole magnet, $C_{n}(n \neq 3) / C_{3} \ll 1$, when the $C R$ is displaced from the magnetic center by Eq. (7), the flux linkages of $Q Q$-coil and QS-coil are calculated from Eq. (8),

$$
\begin{aligned}
& \phi_{Q Q}^{\prime}\left(\theta^{\prime}\right)=-\operatorname{LN}_{Q Q}\left(C_{3} / R^{2}\right) 4 r_{0} r^{2} \sin \left(2 \theta^{\prime}+\theta_{0}-3 \alpha_{3}\right), \\
& \phi_{Q S}^{\prime}(\theta)=-L_{Q S}\left(C_{3} / R^{2}\right)\left(2 \sqrt{2} r^{3} / 3\right) \cos 3\left(\theta^{\prime}-\alpha_{3}\right) .
\end{aligned}
$$

The sextupole field integral is measured from Eq. (15) which does not depend on the position of the location of the cylinder axis. After finding the sextupole strength, the displacement of the $C R$ from the magnetic center is found from Eq. (14).

\section{References}

1. A Radial Coil Probe for Quadrupole Magnet Measurement, S. H. Kim, LS-166.

2. Tangential Winding Coil Probes for Dipole, Quadrupole and Sextupole Magnet Measurements, S. H. Kim, LS-167. 

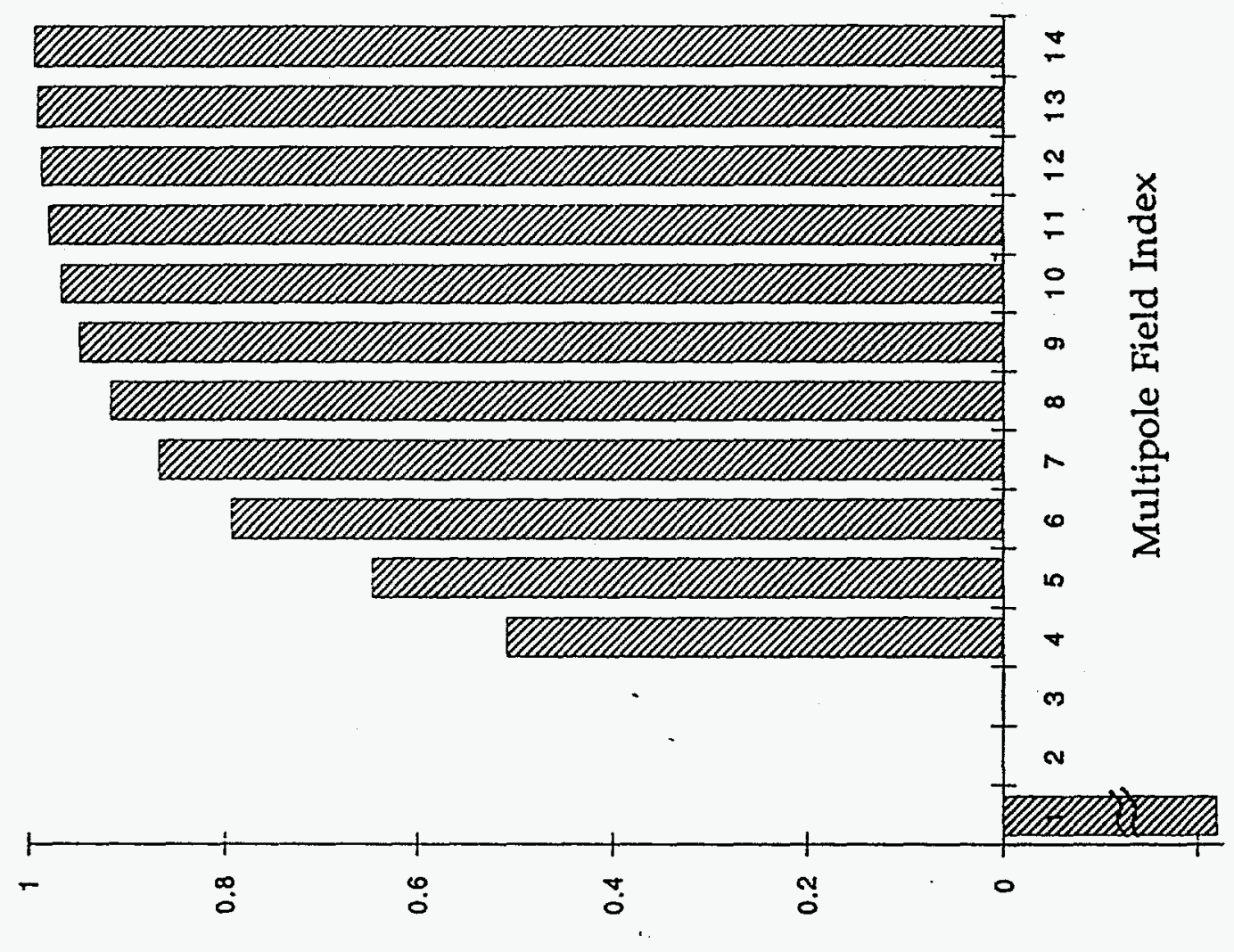

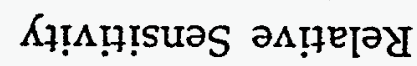

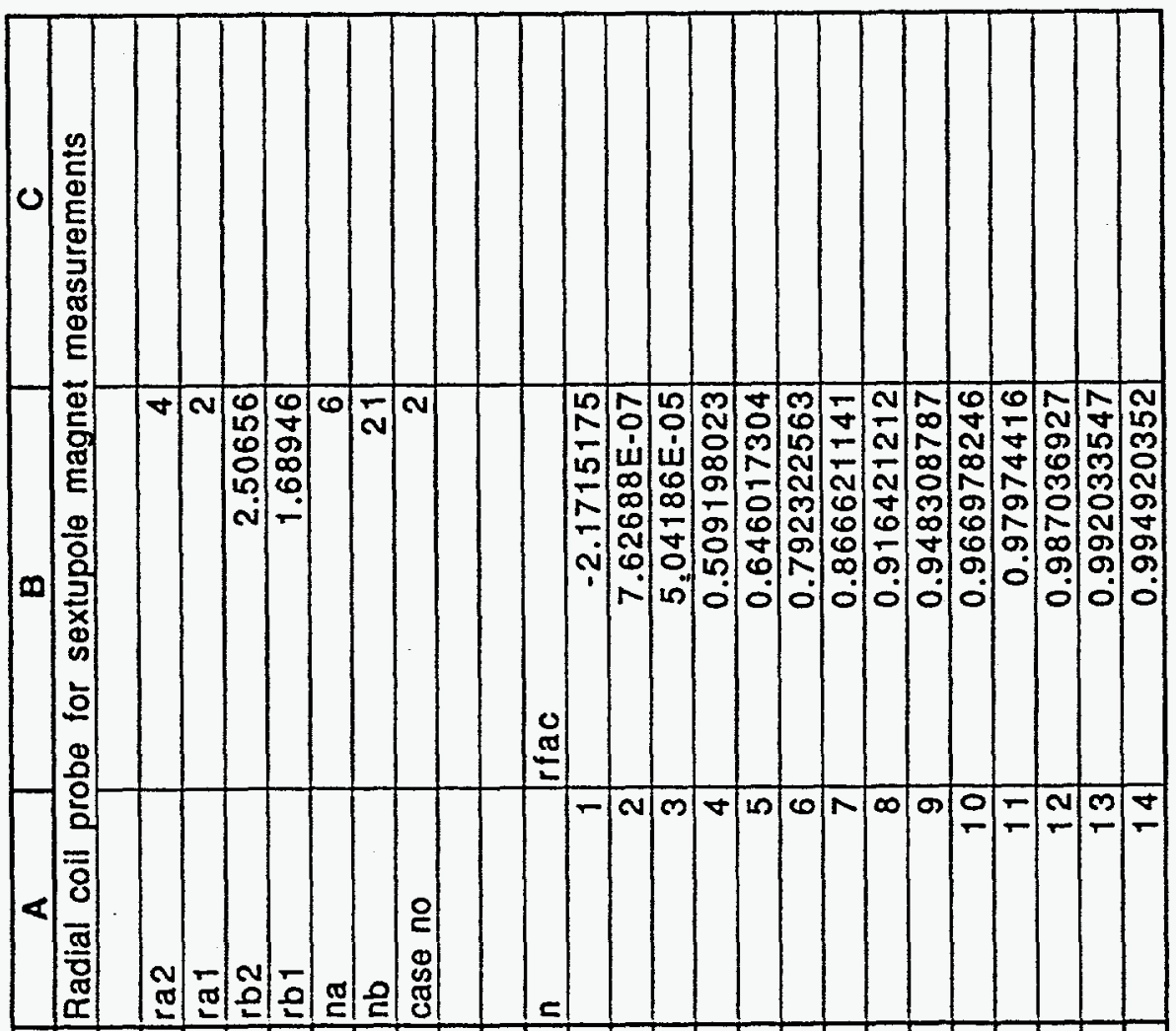

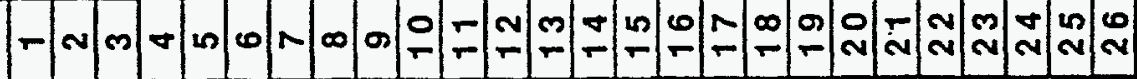


Table 2. Tangential winding coil parameters for sextupole magnet measurements. $\mathrm{N}_{\Delta}=14, \mathrm{~N}_{\mathrm{QQ}}=2, \mathrm{~N}_{\mathrm{QS}}=4, \Delta=15.3882^{\circ}$.

\begin{tabular}{|c|r|r|r|}
\hline & \multicolumn{2}{|c|}{ A } & \multicolumn{1}{c|}{ B } \\
\hline 1 & Tangential coil probe for sextupole magnet measurements \\
\hline 2 & & 14 & \\
\hline 3 & ndelta & 2 & \\
\hline 4 & ngq & 4 & \\
\hline 5 & ngs & 15.3882 & \\
\hline 6 & delta & 3.14159265 & \\
\hline 7 & pi & 4 & \\
\hline 8 & case no & & \\
\hline 9 & & & \\
\hline 10 & $n$ & sensitivity & \\
\hline 11 & 1 & -0.270176879 & \\
\hline 12 & 2 & -0.020356728 & \\
\hline 13 & 3 & -0.012008076 & \\
\hline 14 & 4 & 0.511689016 & \\
\hline 15 & 5 & 1.026172626 & \\
\hline 16 & 6 & 1.007046737 & \\
\hline 17 & 7 & 1.211625989 & \\
\hline 18 & 8 & 0.879256459 & \\
\hline 19 & 9 & 0.531055024 & \\
\hline 20 & 10 & 0.68842362 & \\
\hline 21 & 11 & 0.591558408 & \\
\hline 22 & 12 & 0.99917381 & \\
\hline 23 & 13 & 1.388798069 & \\
\hline 24 & 14 & 1.238283393 & \\
\hline 25 & 15 & 1.307310206 & \\
\hline
\end{tabular}

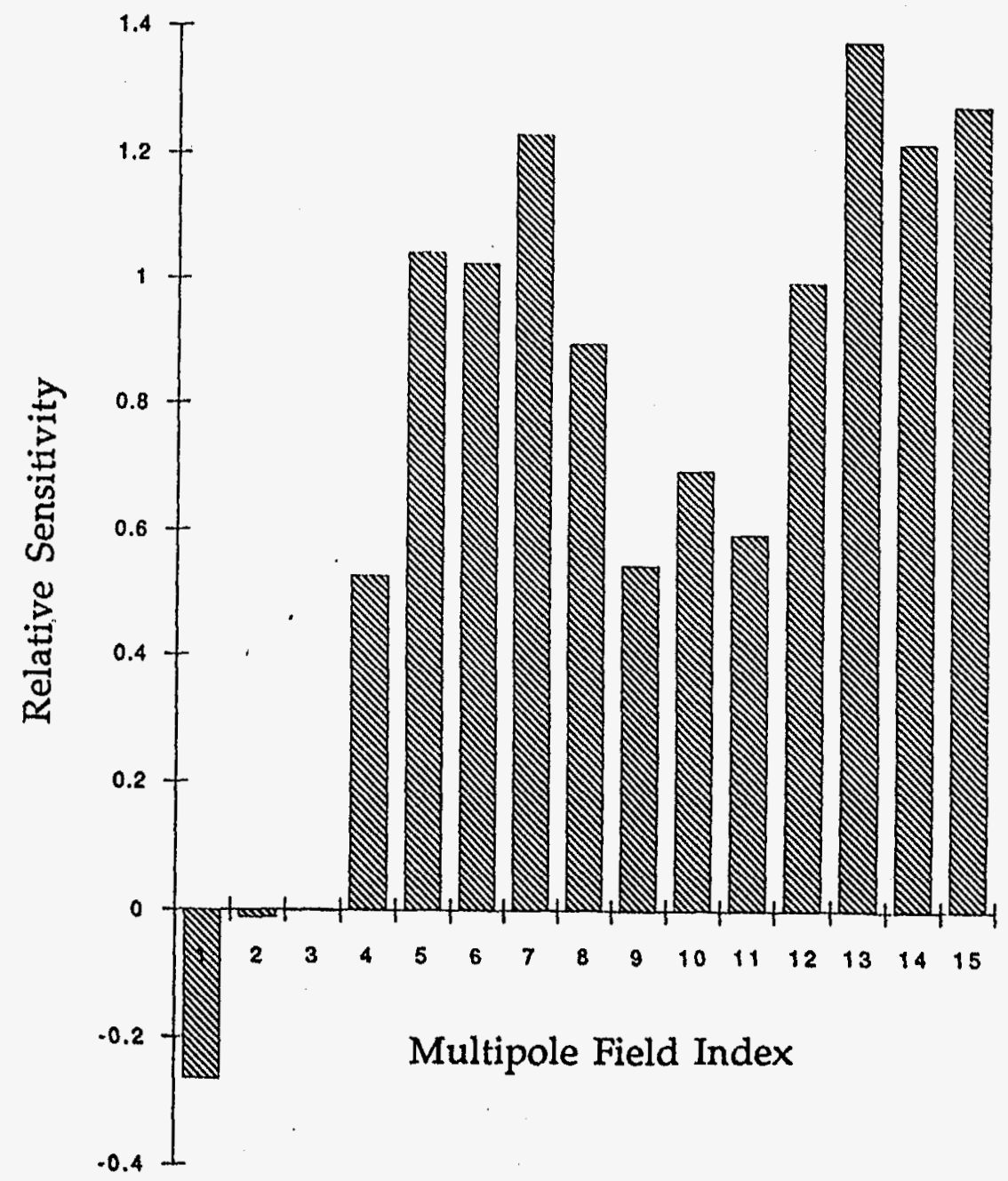




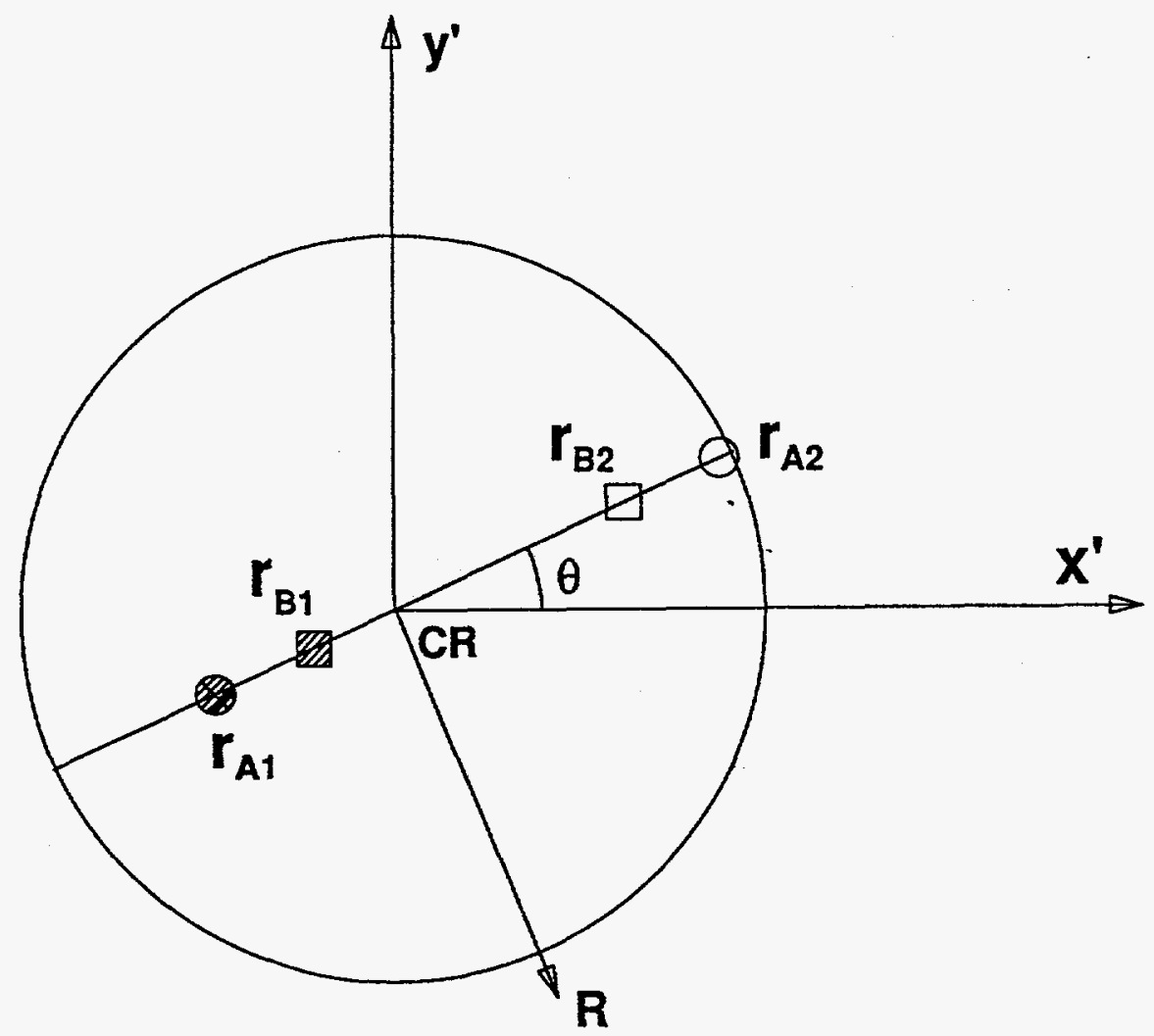

Fig. 1. Cross section of two sets of radial winding coils in a cylinder. Coil $A$ is located at $\left(r_{A 2}, \theta\right)$ and $\left(r_{A 1}, \theta+\pi\right)$, and has a number of turns $N_{A}$. Coil $B$ is located at $\left(r_{B 2}, \theta\right)$ and $\left(r_{B 1}, \theta+\pi\right)$ with $N_{B}$ turns. The cylinder rotation axis (CR) is located at the origin of the $x^{\prime} y^{\prime}$-coordinate system. The CR is in the plane of the two coils. The radius of the measuring magnet aperture can be chosen as the reference radius $R$. The reference angular position, $\theta=0$, is the direction of the $C R / r_{A 2}$. 


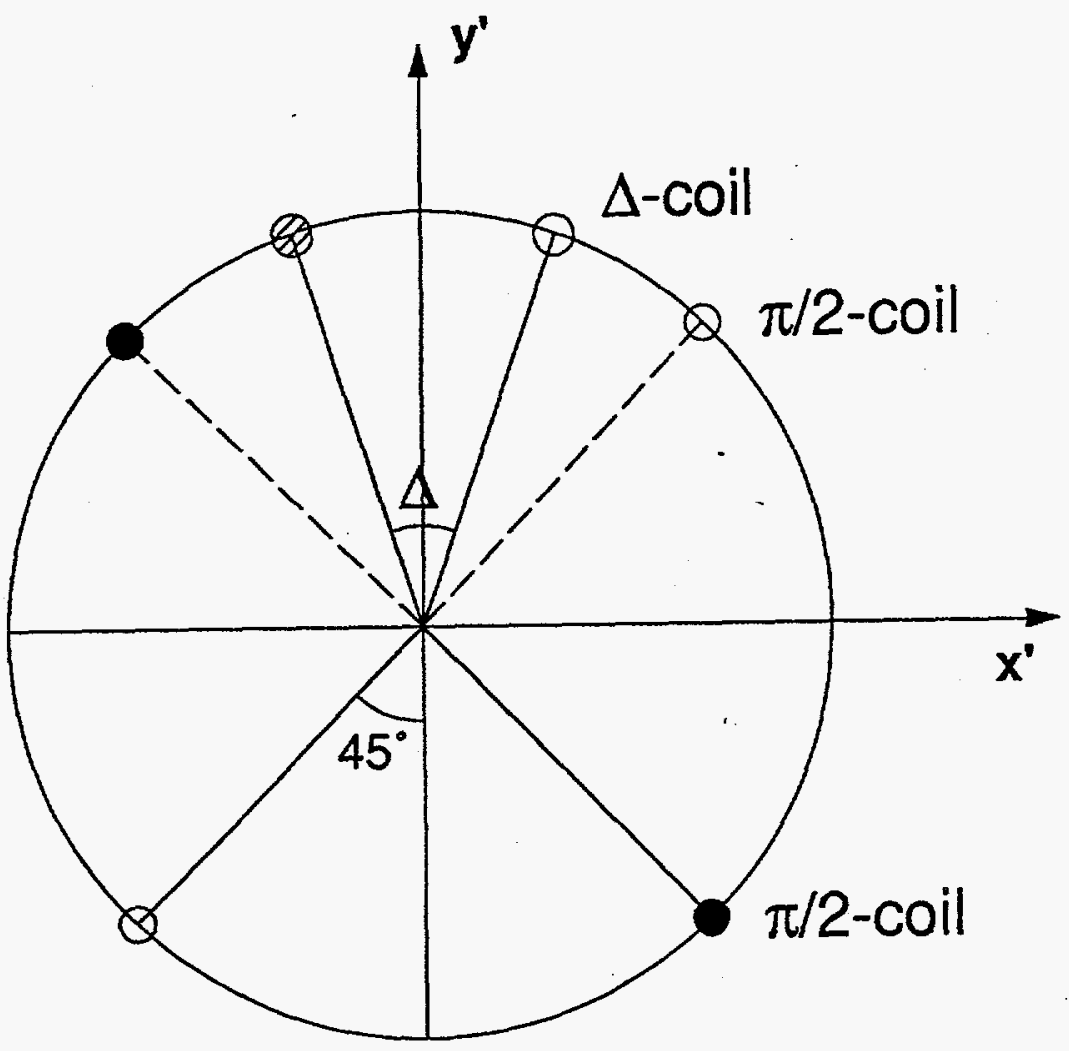

Fig.2. Cross section of a tangential winding coil for sextupole magnet measurements. It consists of one $\Delta$-coil and two $\pi / 2$-coils which are used for the rejection of the quadrupole field for the measurements of the magnetic center, and for the rejection of the sextupole field for harmonic analysis. The two $\pi / 2$-coils, one in the $+y$-plane and one in the $-y$-plane, are connected as shown in the second term of Eq. (11). The two $\pi / 2$-coils are also used for the measurements of the sextupole field integral by connecting them as shown in the third term of Eq. (11). The $x^{\prime}$-axis is the reference angular position, $\theta=0$. 


\section{DISCLAIMER}

This report was prepared as an account of work sponsored by an agency of the United States Government. Neither the United States Government nor any agency thereof, nor any of their employees, makes any warranty, express or implied, or assumes any legal liability or responsibility for the accuracy, completeness, or usefulness of any information, apparatus, product, or process disclosed, or represents that its use would not infringe privately owned rights. Reference herein to any specific commercial product, process, or service by trade name, trademark, manufacturer, or otherwise does not necessarily constitute or imply its endorsement, recommendation, or favoring by the United States Government or any agency thereof. The views and opinions of authors expressed herein do not necessarily state or reflect those of the United States Government or any agency thereof. 